\title{
Bilingual Stroop in English Speakers with Russian as a Second Language: Exploring the Model of the Bilingual Mind
}

\author{
Aleksandra Bril \\ Pitzer College, Class of 2013 \\ Rebecca Green \\ Harvey Mudd College, Class of 2011
}

\begin{abstract}
This report describes an investigation of a bilingual model of the mind and whether or not bilingual speakers are able to ignore one language while accessing another. English Speakers who had been formally studying Russian for at least two years and monolinguals with no previous Russian training were tested with an English Stroop test, a cross-language Russian Stroop test, and an English Control. While the monolinguals displayed no interference during the Russian Stroop task and significant interference during the English Stroop task, bilinguals showed as much interference for the Russian Stroop task as they did for the English one. This suggests that bilinguals unconsciously access both languages simultaneously.
\end{abstract}

\section{Introduction}

Being able to speak multiple languages is an impressive skill. But how do bilinguals keep from combining languages while they speak? Do they have the ability to repress one language completely? This study is concerned with how the bilingual mind stores and accesses language, and whether it has a "switch" that allows bilinguals to go consciously back and forth between languages. Current models of the bilingual mind include two levels: on one level is the lexicon, which is separate for each language; this lexical level connects to another level which contains general concepts, grammar, and semantic knowledge (Sumiya and Healy, 2007). The various models share the notion that each language's lexicon is stored separately, but they disagree on the degree of shared higher-level knowledge in the second level. For example, Ullman's model assumes grammar is stored separately for each language, while other models, such as Green's model, assume that grammar for all languages is shared in one region of the mind (Kciuk, 2009). Our experiment was designed to determine the extent that a bilingual can shut off access to their semantic knowledge, either in their native or learned language, by using a variation of the Stroop test.

The Stroop test, developed and published by J.R. Stroop in 1935, measures the response times for reading congruent and incongruent colored words. The Stroop task requires participants to announce the colors of the words presented. The given words are color words like red, green, blue, or purple, printed in an incongruent color. For example, the word red could be 
printed in green ink. When participants are asked to report on the color of the word, they find it difficult to ignore the incongruent color word. The experiment shows that people read words even when they consciously try not to, activating the semantic concept represented by the word, which conflicts with the semantic concept of the color of the ink. This conflict results in a significant delay in the reader's identifying and announcing the color of the word, and the degree of conflict is measurable by comparing the time it takes for the reader to announce the color of an incongruent color word versus a congruent or neutral word. This confirms the separation of lexical and semantic knowledge in the mind, because without it, we would not expect to see any semantic interference from the printed word.

A study done by Roelofs (2009) used the Stroop to test native Dutch speakers with English as a second language. These subjects reported proficiency and fluency in both languages. The participants in this experiment were shown incongruent color words in English (e.g. "red" printed in green ink) and asked to name the color of the printed word in Dutch. They were also shown incongruent color words in Dutch and asked to name the color of the printed word in Dutch. As a control, they were also asked to name the colors of a set of printed Xs and Vs. The researchers found that participants took the same amount of time to complete the Dutch Stroop task and the cross-language English Stroop task. Stroop interference was prevalent in both the native language and the learned language, suggesting that the bilingual speaker has equal access in words that appear in both the Dutch and English lexicon. Inspired by Roelofs' study, the experiment my partner and I performed used Russian students to investigate how language is stored, and specifically whether or not bilingual speakers can "turn off" one language while switching and accessing another. If such a switch existed, it would indicate that bilinguals can consciously avoid stimulating the lexical areas of the mind, reducing the amount of Stroop interference and possibly reducing the effect of other types of cognitive dissonance. By comparing the times it took for the participants to complete the tasks, we attempted to determine if our hypothesis was correct: Bilinguals can turn off one language when accessing another.

\section{Method}

We tested 25 people, 12 of whom were bilingual, with an English Stroop, cross-language Russian Stroop, and English control and we compared the response times of bilingual and monolingual participants.

Participants: Participants were between the ages of 18 and 23 and attended one of the Claremont Colleges. They were found by responding to email advertisements requesting Russian speakers. The test subjects were 12 participants who had been formally studying Russian for at least two years but were not native speakers. The control group was 13 students who had no previous in-classroom exposure to Russian and were not native speakers.

Materials: Subjects completed a questionnaire that asked whether or not they were a native speaker and how long they had been formally studying Russian. Next they were presented with the words "red, blue, green, yellow" in Russian and asked if they could read and understand 
them. The third sheet of paper contained the English Stroop test which showed four columns of ten color words in each column: red, green, blue, and yellow printed in incongruent ink (e.g. "red" was printed in yellow ink). The next sheet contained a Russian Stroop test which had four columns of ten color words in each column: red, blue, green, and yellow written in Russian orthography and printed in incongruent ink (е.g. "синий” (Russian for blue) was printed in green ink). The next test was the control test, which included four columns and ten rows of neutral, non-color related words in English, which were printed in either red, blue, yellow, or green. On each test, the distribution of the color words along with the color that they were printed in was random.

Procedure: After completing the questionnaire and saying whether or not they were able to read and understand the Russian orthography for the colors red, blue, green, and yellow, the participants were given the English Stroop, Russian Stroop and English Control tests. They were instructed to read down the columns and name all of the colors of the printed words in English as fast as they could. First they were presented with the English Stroop, then the (crosslanguage) Russian Stroop, and finally the English Control. An online stopwatch was used to see how long it would take participants to name the colors of the words. The timer started as they named the color of the first word on the sheet and stopped after they finished naming the color of the last word on the sheet.

\section{Results}

Figure I shows that, on average, monolinguals were able to complete the Russian Stroop task approximately twelve seconds faster than they were able to complete the English Stroop task and they were able to complete the English Control approximately eight seconds faster than they were able to complete the English Stroop. Both monolinguals and bilinguals took the same time to complete the English control, with a mean difference of less than a second between the groups. For bilinguals, there was only approximately a one-second mean difference in the time it took to complete the Russian Stroop and the English Stroop.

\section{Discussion}

Our hypothesis was incorrect: bilinguals cannot turn off one language when accessing another. In our experiment, monolinguals were faster in completing the cross-language Russian Stroop task than bilinguals were. This shows that there was no inter-language interference for monolinguals, which is to be expected, as there was no lexical representation for the Russian words in the monolingual mind, and thus no semantic interference would have occurred.

For bilinguals, however, the mean response times on Russian Stroop and English Stroop tasks were not significantly different, showing that it takes bilinguals approximately the same amount of time to access the Russian and English words for colors and resolve the subsequent semantic conflict. Unlike the monolinguals, when shown a Russian word for a color in an incongruent color, the bilingual speakers were unable to ignore the semantic concepts 
represented by the Russian words and just say the color in English. This significant difference in interference when completing the Russian Stroop task shows that bilinguals cannot avoid reading words and accessing the relevant semantic knowledge for any language they know. This disproves our hypothesis concerning a language switch, since such a switch would give the bilinguals the ability to turn off their access to the lexical knowledge for the language(s) they are not using.

The results in our study are congruent with those of Roelofs (2009) with one major exception. The participants in Roelofs's study reported fluency in their second language, whereas none of the participants in our study did. The majority of the subjects in our study had been formally studying Russian for only two years. However, interlanguage interference was still prevalent. This interference leads us to infer that languages can be accessed simultaneously even if a completely fluent system does not exist as part of the mind's language model. This supports the theory that semantic knowledge is not stored separately for each language a person speaks, but instead is stored together and subject to interference even when the lexical representations are distinct.

Figure I: Mean time for completion of tasks

\begin{tabular}{|l|l|l|}
\hline & Monolinguals & Bilinguals \\
\hline Russian Stroop & $26.787 \mathrm{sec}$ & $36.648 \mathrm{sec}$ \\
\hline English Stroop & $38.763 \mathrm{sec}$ & $35.241 \mathrm{sec}$ \\
\hline English Control & $30.299 \mathrm{sec}$ & \\
\hline & & $29.522 \mathrm{sec}$ \\
\hline
\end{tabular}




\section{References}

Kciuk, O. (2009), "The bilingual brain: understanding the neural bases of language representation and control." University of Toronto Journal of Undergraduate Life Science, Vol. 3 No. $176-78$

Roelofs, A. (2009), "Attention and Facilitation: Converging Information Versus Inadvertent Reading in Stroop Task Performance.” Journal of Experimental Psychology: Learning, Memory, and Cognition, Vol. 36, No. 2, 411-422

Sumiya, H. \& Healy, A. (2007), "The Stroop Effect in English-Japanese Bilinguals: The Effect of Phonological Similarity.” Experimental Psychology 2008; Vol. 55, No. 2: 93-101 
